\title{
9. Maximilien Aue: une homosexualité de rigueur?
}

\section{Éric Levéel}

Il serait vain de réitérer combien le lien entre la sexualité et le Mal a été exploité en littérature, au théâtre et dans les œuvres filmiques. Il serait vain de nier combien la «souillure» homosexuelle s'agrippe à l'iconographie et à l'historiographie du nazisme, combien la culture masculine de cette idéologie demeure un terreau fertile pour des relations «coupables» au sein du nouveau Volk qu'elle tente de créer, de propager et de renforcer.

Le nazi homosexuel, sexuellement pervers et/ ou sadomasochiste fait tant partie d'une certaine imagerie collective que l'on peut se demander si Jonathan Littell en créant le personnage-narrateur de son roman Les Bienveillantes sous les traits d'un esthète homosexuel ne s'est pas contenté de reproduire un schéma développé avant lui par Luchino Visconti dans Les Damnés (1969) ou bien par Pier-Paolo Pasolini dans Salo ou les 120 journées de Sodome (1976). Littell aurait-il voulu «noircir» plus encore un personnage dont les seules actions et les seules prises de position auraient aisément suffi à déclencher le dégoût du lecteur devant une telle représentation de ce Mal ordinaire - pour reprendre le vocable d'Hannah Arendt -, de ce Mal absolu. Serions-nous alors devant une homosexualité de rigueur, une «tare» supplémentaire garantissant au lecteur la description d'un cloaque privé tout aussi nauséabond que la fange nazie: l'homosexualité comme un écho pathologique à une idéologie, à ses manifestations et à sa structure même. On retrouverait par conséquent la thématique viscontienne et pasolinienne du pervers polymorphe revêtu de l'uniforme ss ou fasciste. On s'éloignerait aussi de la vision merlienne de La Mort est mon métier dans laquelle le personnage de Rudolf Lang se trouve être, quant à lui, un père de famille ordinaire, un exécutant loyal et obéissant, le rouage d'une machine mortifère, sans pathologies apparentes pouvant expliquer sa fonction de 


\section{2 Éric Levéel}

bourreau suprême au sein du camp d'Auschwitz, si ce n'est une rigidité d'esprit héritée d'une éducation catholique stricte administrée par un père omniprésent et omnipotent - Le Führer devenant ainsi le prolongement évident du père pour Rudolf Lang. Si les références au roman de Robert Merle ont fusé dès la publication des Bienveillantes, si Jonathan Littell a admis à plusieurs reprises dans la presse l'avoir lu attentivement, si les deux auteurs ont pour personnages principaux les bourreaux plutôt que les victimes vues comme des masses plus ou moins informes - quelques exceptions notoires apparaissent néanmoins dans l'ouvrage de Littell -, la comparaison semble s'arrêter là tant Rudolf Lang et Maximilien Aue sont antinomiques, tant la démarche de Littell est divergente de celle plus historique, plus documentaire de Robert Merle qui se contente de transcrire plus ou mois fidèlement les comptes-rendus du procès de Rudolf Höß à Nuremberg et les entrevues avec le psychiatre commis d'office, le tout de manière romancée. Chez Littell, outre la différence fondamentale entre Aue et Lang, il s'agit d'une véritable œuvre de fiction, Maximilien Aue pouvant, il nous semble, s'apparenter à de nombreux bureaucrates éduqués de la ss et à personne en particulier dans ce que son personnage possède les caractéristiques d'un nazi convaincu sans tomber pour autant dans la caricature. Car tel est l'intérêt de la créature littellienne elle-même: l'inattendu, et l'outrance de cet inattendu. La vie privée de Maximilien Aue est inattendue car là où l'homosexualité n'aurait pu être qu'une preuve supplémentaire de la dégénérescence du personnage, une pathologie annexe selon une grille aux relents d'homophobie ou bien la confirmation d'une dégradation morale, ou tout du moins psychologique de par le caractère «marginal» de cette homosexualité qui n'en est intrinsèquement peut-être pas une. Comme nous l'avons mentionné, Maximilien Aue n'est pas un père de famille tranquille comme Rudolf Lang retrouvant sa femme et ses enfant une fois son devoir d'exterminateur accompli; au contraire, sa vie privée ne lui offre pas le luxe d'effectuer une transition entre l'anormalité et la normalité - si tenté que ces deux termes aient encore un sens dans l'œuvre de Jonathan Littell - non pas parce que Maximilien est homosexuel mais simplement parce que son homosexualité est fondamentalement marginale, réfléchissant «l'anormalité» de sa relation avec sa sœur jumelle Una, Baronne von Üxküll. Elle peut être considérée comme marginale non pas dans ce que certains comprendraient comme une perversion en ellemême mais parce qu'étrangement cette homosexualité pratique traduit la complexité d'un amour véritable et absolu pour une femme: Una, l'unique. 
L'homosexualité de Maximilien Aue - appelons-la ainsi - ne représente pas le duo classique de la sexualité et du Mal fondé sur un rapport de force maître-esclave; à l'inverse, l'officier Aue ne trouve aucun plaisir pervers dans la subjugation sexuelle d'êtres dits inférieurs, comme d'autres officiers. Il ne pense pas aux Juifs, aux Polonais, aux prisonniers comme à des objets sexuels que son pouvoir lui permettrait de dominer et d'humilier. Ses partenaires sexuels sont soit des connaissances (d'autres officiers ss et un diplomate roumain) soit des prostitués parisiens. Loin de vouloir dominer ses «conquêtes», il désire l'être par leur phallus, sa passivité sexuelle allant à l'encontre de l'image masculine et guerrière.

Jonathan Littell ne nous épargne aucun détail de chaque rencontre sexuelle dont deux dans le Paris de l'Occupation offrent au lecteur une compréhension totale de ce que nous avons voulu appeler homosexualité marginale, c'est-à-dire une homosexualité dont les racines ne se trouvent pas dans une attraction réelle pour un autre homme et dont le choix de la passivité sexuelle est un choix érotique, mais une homosexualité profondément ancrée dans le désir d'être autre, ou dans ce cas précis dans la volonté de s'identifier, de ne faire qu'un avec l'être aimé: Una. Avant et pendant la puberté, Maximilien et sa sœur ont eu une relation incestueuse qu'il ne voit pas ainsi mais plutôt comme un moyen de consolider sa gémellité, l'unicité de leur lien; la pureté et l'intensité de l'amour que Maximilien ressent et sa destruction par leur mère lorsque leur relation «immorale» est découverte, vont déterminer et définir ses comportements sexuels. Exilé dans un pensionnat catholique strict et pervers - répétition prophétique, il nous semble, des camps d'extermination dont il deviendra inspecteur - dans lequel il découvre les pratiques homosexuelles avec des garçons plus âgés qu'il reproduit seul rentré chez lui, pensant qu'être pénétré par d'autres ou par divers objets masturbateurs lui permet de comprendre ce que ressent une femme, plus précisément ce qu'Una avait ressenti, tentant ainsi de dépasser l'identification pour devenir elle par le biais d'une sodomie qui reniant l'anal devient vaginale. On pourra néanmoins s'interroger sur l'aspect pathologique d'un tel cheminement, sur ce que Julia Kristeva, en parfaite psychanalyste, nomme «son désir morbide de prendre la place du sexe féminin $[\ldots]^{1} \gg$. Il est certes possible d'analyser son désir de ne faire qu'un avec sa sœur de la sorte mais il nous

1 Julia Kristeva, «De l'abjection à la banalité du mal», dahns http://www.kristeva.fr, Julia_Kristeva/791450DF-6D50-4A2E-ACB7-Ec98BF7E1D11.httml, conférence avec Jonathan Littell, auteur du roman Les Bienveillantes, invité par le Centre Roland Barthes (Université Paris VII), à l'ENS, le mardi 24 avril 2007. 


\section{4 Éric Levéel}

semble également qu'en reléguant la psychanalyse, et le jugement moral, à la périphérie de notre étude, nous sommes à même d'apporter un nouvel éclairage sur l'homosexualité marginale de Maximilien Aue.

Nous retrouvons cet élan de féminisation très tôt dans le roman bien avant que le lecteur ne soit directement confronté aux impulsions incestueuses et à l'amour possessif et entier de Maximilien pour Una qui «l'abandonnera» aux portes de l'âge adulte lors de ses études de psychologie jungienne à Zurich. Dès le chapitre d'introduction intitulé Toccata le narrateur encore inconnu affirme: «[...] La réalité, je ne rougis pas de le dire, c'est que j'aurais sans doute préféré être une femme. Pas nécessairement une vivante et agissante dans ce monde, une épouse, une mère; non, une femme nue, sur le dos, les jambes écartées, écrasée sous le poids d'un homme, agrippée à lui et percée par lui, noyée en lui en devenant la mer sans limites dans laquelle lui-même se noie, plaisir sans fin, et sans début aussi ${ }^{2} \gg$. Cette femme soumise, d'essence purement vaginale, sans existence propre en dehors de son désir érotique, c'est à la fois Maximilien qui couche avec des hommes qu'il méprise et qui le pénètrent violemment mais c'est bien évidemment Una assujettie au désir déferlant de son frère comme ce dernier aurait voulu qu'elle soit pour l'éternité. Comme un écho à la femme aimée "plus que tout au monde ${ }^{3}$ », il nous faut rapprocher le passage précédent avec celui advenant lors du séjour de Maximilien Aue dans le Paris occupé par les forces allemandes. Après une visite au Grand Palais où il a longuement admiré un Apollon citharède de Pompéi, il se dirige vers Pigalle et entre dans un bar spécialisé dans les rencontres masculines, y trouvant rapidement une jeune frappe qu'il ramène à son hôtel. Ce qui ne pourrait être que la description crue d'une relation sexuelle entre deux hommes, se transforme en un nouvel hymne à l'être aimé vers lequel tend toute une existence:

Pour lui, mon cul s'ouvrit comme une fleur, et lorsque enfin il m'enfila, une boule de lumière blanche se mit à grandir à la base de mon épine dorsale, remonta lentement mon dos, et annula ma tête. Et ce soir-là, plus que jamais il me semblait que je répondais ainsi directement à ma sœur, me l'incorporant, qu'elle l'acceptât ou non. Ce qui se passait dans mon corps, sous les mains et la verge de ce garçon inconnu, me bouleversait. Lorsque ce fut fini, je le renvoyai mais je ne m'endormis pas, je restai couché là sur les draps froissés, nu et étalé comme un gosse anéanti de bonheur. ${ }^{4}$

2 Les Bienveillantes, Paris, Gallimard, 2006, p. 29.

3 Ibidem, p. 29.

4 Ibidem, pp. 462-463. 
Bonheur d'avoir pu un bref instant anéantir sa réalité, «annuler» son identité pour adopter entièrement, lui semble-t-il, celle de sa sœur qui l'a rejeté définitivement en épousant l'aristocrate poméranien von Üxküll. Cette identification totale avec sa jumelle a jailli de l'absence, de la «trahison» et du manque constant de l'autre: de la séparation exigée par la mère et finalement agrée dans les faits par la sœur.

L'identification avec Una va encore plus loin dans les derniers mois de la guerre lorsque Maximilien passe dix jours dans le plus complet isolement sur le domaine de sa sœur et de son mari en Poméranie, séjour durant lequel il va donner libre court à sa quête impossible en oscillant entre un Orlando woolfien - «la drag queen insolemment pathétique» selon Julia Kristeva ${ }^{5}$ traversant le temps intérieur du souvenir et des fantasmes et un Tristan médiéval attendant, en vain, sa promise interdite: «j'aurais voulu couper des branches, construire une hutte que j'aurais tapissée de mousse, et y passer la nuit, nu; mais il faisait quand même trop froid, et puis il n'y avait pas $\mathrm{d}^{\prime}$ Yseut pour la partager avec moi $[\ldots]^{6} \gg$ - il va sans dire que les deux jumeaux d'Una portent des prénoms qui en appellent mimétiquement à leur père présumé, mais jamais nommé: Maximilien. Une fois encore dans le roman, l'effet de miroir et de réflexion constante s'opère, ainsi qu'une ironie évidente qui parcourt le récit ${ }^{7}$.

Dans le chapitre intitulé Air, auquel nous venons de faire référence sans le nommer, il n'est plus question d'homosexualité, l'autoérotisme du protagoniste ne s'alimente aucunement de fantasmes masculins mais, bien au contraire, d'images purement sororales, aussi dérangeantes soient-elles. C'est que l'homosexualité de Maximilien Aue est à la fois omniprésente et introuvable. Omniprésente de par sa manifestation purement sexuelle d'adulte dans le roman et introuvable dans sa définition identitaire et psychologique. Johanna Lehr et Daniel Lehr y voient une homosexualité perverse, «élément central $\mathrm{d}[\mathrm{u}]$ livre $^{8}$ ». Il existe néanmoins une contradiction de taille avec cette définition puisque Jonathan Littell lui-même rejette l'étiquette - ce que les deux universitaires soulignent également - ; lorsque Daniel Cohn-Bendit, lors d'un débat au Berliner Ensemble à l'occasion de la publication de la traduction du roman en allemand, affirme que Max

5 Julia Kristeva, «De l'abjection à la banalité du mal», art. cit.

6 Les Bienveillantes, op.cit., p. 826.

7 Cf. Annick Jauer, Université de Provence «Ironie et génocide dans Les Bienveillantes de Jonathan Littell», dans http://www.fabula.org/colloques/document982.php.

8 Cf.http://www.relatio-europe.eu/la-revue/86-etudes/3802--les-bienveillantesde-jonathan-littell--histoire-dl-une-bevue 


\section{6 Éric Levéel}

Aue est «un intellectuel très cultivé et homosexuel», Littell lui répond sans hésitation: «vous êtes sûr...? ${ }^{9} »$. Julia Kristeva, quant à elle, décèle la «féminisation victimaire de cette homosexualité passive ${ }^{10}$ »; si féminisation il y a sans aucun doute, si elle est victimaire car vécue dans l'absence et le manque, peut-on encore la catégoriser comme homosexualité par le simple fait de la sodomie passive qui ne constitue pas ici, il nous semble, un choix érotique, mais une communion, une fusion, un mimétisme avec l'Unique aimée. Il demeure évident que cette quête est inexorablement vouée à l'échec, ce qui la rend pathologique dans ce qu'elle recèle de souffrance et d'inassouvi. Si perversion il y a dans la sphère privée, c'est celle faite à soi-même dans cette course effrénée vers l'inaccessible et l'absurde: Maximilien Aue n'est pas un Sisyphe heureux de rouler inlassablement sa pierre, bien au contraire comme Caligula il désire la lune et retrouver sa sœur pour l'éternité - il est à noter que si Una ne meurt pas physiquement, elle disparaît symboliquement du récit à la fin du chapitre Air alors que le monde public de son frère s'effondre devant la poussée des troupes soviétiques vers la Poméranie, et enfin vers Berlin bombardée et exsangue. Chronologiquement parlant, Una ne disparaît jamais puisqu'elle est l'inconnue du chapitre d'introduction - cette épitaphe qui ne s'excuse de rien - mais elle a été reléguée au néant, éclipsée par l'Histoire et l'existence «normale» et bourgeoise de son frère.

Maximilien Aue affirme très tôt: «les types avec qui j'ai couché, je n'en ai jamais aimé un seul, je me suis servi d'eux, de leur corps, $c^{\prime}$ est tout ${ }^{11}{ }^{1}$. Ces hommes de passage n'ont pour seule fonction que de le pénétrer et de l'aider ainsi à communier, transcendé, avec Una. Si le roman est parcouru des pensées érotiques et amoureuses du personnage principal à l'égard de sa sœur, culminant en Poméranie dans ce que Kristava nomme «une fureur d'auto-pénétration érotique ${ }^{12}{ }^{2}$, peu de scènes homosexuelles avec d'autres hommes y sont présentes. Une première dans le Tiergarten de Berlin - lieu notoire de rencontres homosexuelles, mais également lieu où tout commence et finit pour l'officier ss Aue; il s'agit aussi de l'espace de naissance des Furies et de leur assagissement en Bienveillantes - va décider de l'avenir professionnel du héros qui, arrêté par la police au soupçon d'un délit lié au paragraphe 175 du Code pénal allemand, se voit secouru par

9 Daniel Cohn-Bendit, «Jonathan Littell: Les Bienveillantes, l'Allemagne et sa mémoire», Le Figaro, 3 mars 2008.

10 Julia Kristeva, «De l'abjection à la banalité du mal», art.cit.

11 Les Bienveillantes, op.cit., p. 29.

12 Julia Kristeva, «De l'abjection à la banalité du mal», art. cit. 
Thomas Hauser et «contraint» de rejoindre le Service de sécurité (sD) de la ss, grande ironie du roman s'il en est une...

Quant à la relation avec l'officier Partenau dans le Caucase, elle annonce celle, fort brève, avec la petite frappe parisienne que nous avons présentée précédemment. Pour s'en convaincre, il nous suffit de lire le passage suivant en nous remémorant celui des pages 462 et 463:

Au début, lorsque ça entre, c'est parfois difficile, surtout si c'est un peu sec. Mais une fois dedans, ah, c'est bon, vous ne pouvez pas vous imaginer. Le dos se creuse et c'est comme une coulée bleue et lumineuse de plomb fondu qui vous emplit le bassin et remonte lentement la moelle pour vous saisir la tête et l'effacer. Cet effet remarquable serait dû, parait-il, au contact de l'organe pénétrant avec la prostate, ce clitoris du pauvre, qui chez le pénétré se trouve contre le grand côlon, alors que chez la femme, si mes notions d'anatomie sont exactes, elle se trouve séparée par une partie de l'appareil reproducteur $[\ldots]]^{13}$

On y retrouve cette idée de boule de lumière qui anéantit toute conscience de soi-même, et Aue y décrit la sodomie en des termes féminins ${ }^{14}$, faisant de sa prostate ce clitoris qui lui manque cruellement. De plus, la douleur ressentit parfois lors de la pénétration anale, c'est celle de la défloraison chaque fois renouvelée, celle ressentit la première fois par Una une fois les jeux enfantins dépassés.

Avant d'en venir à la deuxième rencontre de Paris, il nous semble important de briser l'ordre chronologique pour nous pencher sur la relation que Maximilien va entretenir avec Mihaïle diplomate bisexuel roumain dans les derniers mois avant la chute de Berlin. Cette relation ne suit pas le schéma habituel voulu par Aue qui est «d'éviter les intellectuels ou les hommes de ma classe sociale: ils v[eulent] toujours parler, et [ont] une fâcheuse tendance à tomber amoureux ${ }^{15}$ », ce qui en effet ne peut être compatible avec la quête, aussi folle et absurde soit-elle, du récipiendaire. Mais Mihaï s'érige en symbole, celui d'un monde amoral et moribond, qui va bientôt agoniser sous les bombes alliées et soviétiques tout comme il agonisera lui-même sous le manche à balai pressé contre sa gorge par Maximilien Aue. Mihaï est un divertissement, une impasse amusante qui ne mène nulle part, qui ne rapproche pas d'Una et qui permet d'oublier l'amour silencieux d'Hélène auquel il ne peut répondre ; il s'agit d'une pure diversion comme 13 Les Bienveillantes, op. cit., p. 189. .

14 On pourra considérer que la sodomie est également un moyen de revivre l'expérience d'Una l'année de leurs treize ans lorsque ses règles apparurent. Ibidem, p. 443

15 Ibidem, p. 764. 


\section{8 Éric Levéel}

le souligne Maximilien: «je me servais de lui brutalement, comme pour laver de ma tête les désirs muets de mon amie, ou ma propre ambiguïté ${ }^{16}{ }^{1}$. De manière intéressante, là où on n'a pas épargné au lecteur le détail des rencontres sexuelles masculines du personnage principal, avec Mihaï rien n'est décrit, si ce n'est la phrase sibylline que nous venons de citer, ainsi qu'une brève référence, quelques jours plus tard, à une soirée orgiaque se tenant à la résidence de l'ambassadeur croate ${ }^{17}$. Le jeune diplomate roumain semble être la seule personne à avoir offert à Maximilien Aue un semblant de relation purement homosexuelle dans le sens que leurs rapports ne se soustraient pas à ceux fantasmés par Aue entre lui et sa sœur. Le meurtre de sang-froid de Mihaï ne fait que souligner ce qui pèche dans cette relation stérile placée sous le signe d'une «gaité vaine et frénétique ${ }^{180}$ », à cent lieux de la communion recherchée par Maximilien Aue qui ne peut supporter d'être étiqueté comme homosexuel car il ne l'est fondamentalement pas et qu'avec Mihaï il ne peut se féminiser. Tout comme dans le meurtre de sa mère - meurtre que l'on suspecte, tout comme sa paternité des jumeaux -, Maximilien Aue supprime ce qui fait obstacle, selon lui, à sa quête, à ce qui détermine qui il est réellement.

Cette vision de l'obstacle on la retrouve magnifiée à l'extrême dans la description de la deuxième rencontre parisienne du personnage. Alors que la première lui avait permis d'entièrement se transcender, d'atteindre un certain inaccessible, cette deuxième rencontre qui semble refléter, en apparence, la précédente va se solder par un échec:

Je lui demandai de me prendre debout, appuyé sur la commode, face à l'étroit miroir qui dominait la chambre. Lorsque le plaisir me saisit, je gardai les yeux ouverts, je scrutai mon visage empourpré et hideusement gonflé, cherchant à y voir, vrai visage emplissant mes traits par-derrière, les traits du visage de ma sœur. Mais alors il se passa ceci d'étonnant: entre ces deux visages et leur fusion parfaite vint se glisser, lisse, translucide comme une feuille de verre, un autre visage, le visage aigre et placide de notre mère, infiniment fin mais plus opaque, plus dense que le plus épais des murs. Saisi $\mathrm{d}^{\prime}$ une rage immonde, je rugis et fracassai le miroir d'un coup de poing ${ }^{19}$.

De même qu'avec Mihaï, le narrateur va tenter de supprimer l'obstacle que constitue sa mère honnie, et ceci rapidement après cette scène révélatrice dans laquelle Lady Macbeth ${ }^{20}$ qui peuple ses rêve et empoisonne son souci

16 Ibidem, p. 765.

17 Ibidem, p. 768.

18 Ibidem, p. 765.

19 Ibidem, p. 474.

20 Ibidem, p. 475. 
de transcendance se transformera en Clytemnestre tuée par Oreste. En tuant sa génitrice-obstacle, Aue pense pouvoir recoller les fragments du miroir qu'il vient de briser car sa réflexion, tel un mauvais conte, ne lui renvoyait plus l'image de l'Autre aimée: sa propre image. Mais la mort de la mère ne résout rien puisque la clé appartient à Una qui, devenue adulte, rejette le passé ${ }^{21}$ auquel Max se raccroche comme unique point d'attache humain dans une existence publique inhumaine et monstrueuse. Si la vie privée du narrateur va à l'encontre des lois morales et fait voler en éclats bon nombres de tabous sexuels, il nous paraît possible d'affirmer que son amour pour Una, aussi infantile soit-il, est véritable.

Si le Mal existe, et Maximilien en est un parfait représentant dans le roman, il ne se place pas dans la passion - en ce que la passion peut avoir de pathologique, étymologiquement parlant - d'un frère pour sœur; au lieu du Mal, on y verrait plutôt une maladie incurable sur laquelle aucun contrôle n'est possible. Dans la sphère publique où il n'est pas question de sentiments, le Mal ordinaire s'immisce partout tant le narrateur soutient l'idéologie nazie dans ce qu'elle peut avoir de plus absolue. L'humanité souffrante de Maximilien ne change en rien ses décisions idéologiques, ou pratiques, d'officier du sD et de l'Inspection des camps. Sa fragilité émotionnelle, son amour malheureux n'influencent en rien ses actes publics. On a voulu voir dans ce narrateur «homme-femme ${ }^{22} »$ un être plus sensible à la douleur d'autrui éveillant en lui «de tristes désaccords avec les violences des guerriers $[\ldots]^{23} »$. Son «homosexualité», son hermaphrodisme psychologique, le rendrait donc plus poreux aux horreurs dont il est le témoin. Certes, il désapprouve la violence gratuite de certains de ses collègues vis-à-vis de leurs victimes mais sa «particularité» psychique ne remet absolument pas en cause ce qui régit l'anéantissement des «ennemis» du Reich qui est compris philosophiquement plutôt que politiquement:

Le meurtre des Juifs, au fond, ne sert à rien. Rasch a absolument raison. Ça n'a aucune utilité économique ou politique, ça n'a aucune utilité pratique. $\mathrm{Au}$ contraire, c'est une rupture d'avec le monde de l'économie et de la politique. C'est le gaspillage, la perte pure. C'est tout. Et donc ça ne peut avoir qu'un sens: celui d'un sacrifice définitif, qui nous lie définitivement, nous empêche une fois pour toutes de revenir en arrière. Tu comprends? Avec ça, on sort du monde du pari, plus de marche arrière possible. ${ }^{24}$

21 Ibidem, p. 452.

22 Julia Kristeva, «De l'abjection à la banalité du mal», op. cit.

23 Ibidem.

24 Les Bienveillantes, op. cit., p.137. 
Ce pacte qui va dorénavant lier les exécutants, et le Volk tout entier, c'est ce qui fait de Maximilien Aue ce monstre, non pas son attachement à Una. Son «homosexualité» et sa féminisation ne sont pas des manifestations, des prolongements, de sa monstruosité publique. Sa souffrance personnelle ne développe pas en lui de compassion plus aiguisée pour les victimes: il exécute sa tâche tout en réprouvant, et nous l'avons déjà dit, la violence gratuite et le sadisme, souvent écœuré par la fange sanglante et coprologique qui l'entoure; il ne s'agit pas de compassion mais de dégoût devant les moyens employés.

Jonathan Littell a créé un personnage à la sexualité complexe «qui puisse être lucide, donc détaché, donc distancié par rapport à tous les autres ${ }^{25}$ », un homme qui pense et qui se pense, qui s'interroge sans remettre en cause son engagement idéologique mais en admettant, quelque peu enclin à la mauvaise foi et sans s'excuser, que «son espérance a été déçue, et l'on s'est servi de [sa] sincérité pour accomplir une œuvre qui s'est révélée mauvaise et malsaine ${ }^{26}$ ». Dans cette déclaration de conclusion, mais à la fois d'ouverture du récit romanesque, c'est la sphère publique qui est disséquée, tant il nous semble que celle privée doit être comprise séparément. Là où Maximilien se sent trahi politiquement, dans sa maturité il analyse son amour pour Una comme «la seule chose bonne qu'[il ait] faite ${ }^{27}$ ». Les psychanalystes, à l'instar de Julia Kristeva, y verront sans doute un refus psychopathologique de reconnaître la morbidité d'une telle fixation incestueuse, mais le lecteur ne pourra s'empêcher de croire à cette affirmation malgré la répulsion qu'elle lui inspire en tant que manifestation d'un tabou moral et sociétal. C'est que le peu de «décence» de Maximilien tient dans cette déclaration tant il sait que ses sentiments pour sa sœur ont constitué la seule chose dont il ne soit pas amer ou honteux malgré le rejet, puis l'absence totale, de son Yseut. Il ne nous faut pas oublier également que le narrateur répond à un amour partagé dans sa jeunesse, un amour qui sera interrompu par la mère, qui vivra sa dernière consommation charnelle à Zurich, et qui continuera à exister dans sa conscience grâce à des expédients homosexuels. La pathologie privée réelle de Maximilien Aue c'est le complexe de Peter Pan amoureux de Wendy, mais Wendy grandit et la nursery - le grenier $\mathrm{d}^{\prime}$ Antibes - devient hors limites, alors cesse «L'Âge d'Or ${ }^{28}{ }$.

Grâce, ou plutôt à cause de son amour, pour Una, le narrateur va réaliser

25 Daniel Cohn-Bendit, «Jonathan Littell», art. cit.

26 Les Bienveillantes, op. cit., p.30.

27 Ibidem, p. 29.

28 Ibidem, p. 443. 
l'amplitude du Mal qu'il instille en tant que ss. À la fin du chapitre Air, après avoir simulé sa propre pendaison dans le grenier des von Üxküll lieu érotique récurrent -, il se remémore l'exécution d'une jeune partisane soviétique de Kharkov qui par l'horreur de sa mort reflète l'image de la sœur, de l'Autre humanisé après les milliers d'ombres sans identité dans les fosses et les crématoires:

[...] j'avais vu cette fille que nous avions pendue un jour d'hiver dans le parc derrière la statue de Chevtchenko, une fille jeune et saine et resplendissante de vie, avait-elle joui, elle était très jeune, avait-elle connu cela avant que nous la pendions, de quel droit l'avions-nous pendue, comment pouvait-on pendre cette fille, et je sanglotais sans fin, ravagé par son souvenir, ma Notre-Dame-des-Neiges, ce n'était pas des remords, je n'avais pas de remords, je ne me sentais pas coupable, je ne pensais pas que les choses pu ou dû être autrement, seulement je comprenais ce que voulait dire de pendre une fille, nous l'avions pendu comme un boucher égorge un bœuf, sans passion, parce qu'il fallait le faire [...] c'était une jeune fille qui avait été une petite fille peut-être heureuse et qui entrait alors dans la vie, une vie pleine d'assassins qu'elle n'avait pas su éviter, une fille comme ma sœur en quelque sorte, la sœur de quelqu'un, peut-être, comme moi aussi j'étais frère de quelqu'un, et une telle cruauté n'avait pas de nom, quelle que soit la nécessité objective elle ruinait tout, si l'on pouvait faire ça, pendre une jeune fille comme ça, alors on pouvait tout faire, il n'y avait aucune assurance, ma sœur pouvait un jour pisser gaiement dans un wc et le lendemain se vider en étouffant au bout d'une corde, cela ne rimait absolument à rien, et voilà pourquoi je pleurais, je ne comprenais plus rien, et je voulais être seul pour ne plus rien comprendre. ${ }^{29}$

L'intérêt de ce passage, outre le manque complet de remords de la part $\mathrm{du}$ narrateur qui persiste dans une logique de l'abjection, est que celui-ci associe une victime spécifique à sa sœur et, paradoxalement, dans la mort atroce de celle-ci c'est l'existence même d'Una qui est soulignée, ainsi que sa mortalité. La compassion de Maximilien Aue se porte par procuration sur la personne de sa sœur. En baptisant la jeune suppliciée Notre-Dame-desNeiges, le narrateur lui confère une identité que la corde du bourreau lui avait enlevée; cette jeune Madonne aurait pu être Una si elle n'avait pas eu la chance de naître allemande, mais c'est aussi Maximilien Aue transcendé comme nous le prouve la fin du chapitre alors qu'il «aperçoit» une morte couchée dans la neige du jardin: «[...] je sus alors que le corps de cette fille, que sa nuque tordue, son menton proéminent, ses seins glacés et rongés étaient eux le reflet aveugle non pas, comme je l'avais alors cru d'une image

29 Ibidem, pp. 835-836. 


\section{2 Éric Levéel}

mais de deux, confondues et séparées, l'une debout sur la terrasse et l'autre en bas, couchée dans la neige ${ }^{30}{ }$. Une Sainte Trinité vient de s'établir entre les jumeaux d'Antibes et la morte de Kharkov. En pleurant sur la jeune pendue, Maximilien Aue ne fait que réitérer l'amour fou, et tabou, qu'il porte à Una. Il n'humanise pas le nazisme en sanglotant devant la potence de fortune car il ne pleure aucunement sur son acte et sur les raisons de celui-ci, il reproduit simplement le mécanisme d'identification devant une très jeune femme qui aurait pu être Una, l'unique ancre humaine dans ce marécage infâme de la guerre et de la Solution Finale.

Comme le hurle le narrateur à la fin du chapitre d'introduction, «[...] je suis un homme comme les autres, je suis un homme comme vous. Allons, puisque je vous dis que je suis comme vous! ${ }^{31}{ }^{\prime}$. Comme nous tous sans l'être néanmoins, mais essentiellement humain dans son abjection publique, dans sa capacité aisée au Mal qui, finalement, le déshumanise entièrement. Il s'agit, nous semble-t-il, de la grande ambigüité du récit: la sphère privée si elle rebute contient en elle un ferment universel d'humanité. «L'homosexualité» de Maximilien qui n'aurait pu être qu'un autre trait grossier afin de le noircir encore plus se révèle être non pas un cauchemar freudien mais sa seule attache à la décence incarnée par sa sœur. L'inceste se pose comme unique expérience d'un véritable partage humain, d'un véritable échange. Au milieu de la guerre et des massacres, le souvenir de l'amour incestueux semble être la bouée de quelqu'un qui se noie, consentant, dans l'horreur. C'est la passion incestueuse qui humanise le narrateur, qui l'humanise jusqu'au paroxysme de la folie effleurée en Poméranie.

Maximilien Aue n'est ni un pervers ni un psychopathe ${ }^{32}-c^{\prime}$ est certes un monstre banal dans l'exécution de ses fonctions ss ainsi qu'un matricide pour une question de survie - mais avant tout un homme amoureux d'une femme devenue inaccessible, d'une femme-lumière dont le souvenir fait souffrir autant qu'il réconforte. La soi-disant homosexualité perverse de Maximilien Aue n'est pas de rigueur puisqu'elle n'a en fait aucun lien avec ses fonctions officielles, parce qu'elle ne déborde pas du cadre privé et surtout parce qu'elle en appelle à la vie plutôt qu'à la mort qui l'environne. Pour s'en convaincre, il nous suffit de laisser une dernière fois la parole au narrateur:

$30 \quad$ Ibidem, p. 837.

31 Ibidem, p. 30.

32 Jonathan Littell, «Cosmopolitaine», France Inter, 12 janvier 2006. 
[...] j'essayais de m'imaginer ma sœur les jambes couvertes d'une diarrhée liquide, collante, à la puanteur abominablement douce. Les évacuées décharnées d'Auschwitz, blotties sous leurs couvertures, avaient elles aussi les jambes couvertes de merde, leurs jambes semblables à des bâtons; celles qui s'arrêtaient pour déféquer étaient exécutées, elles étaient obligées de chier en marchant, comme les chevaux. Una couverte de merde aurait été encore plus belle, plus solaire et pure sous cette fange qui ne l'aurait pas touchée, qui aurait été incapable de la souiller. ${ }^{33}$

Una, seule humanité au milieu de l'inhumanité et de la déshumanisation, dernier vestige de l'humanité saccagée de Maximilien Aue.

Université de Stellenbosch - Afrique du Sud

33 Les Bienveillantes, op. cit., pp. 805-806. 